\title{
Axioms for Definability and Full Completeness
}

\author{
Samson Abramsky \\ September 25, 2018
}

\begin{abstract}
Axioms are presented on models of PCF from which full abstraction can be proved. These axioms have been distilled from recent results on definability and full abstraction of game semantics for a number of programming languages. Full completeness for pure simply-typed $\lambda$-calculus is also axiomatized.
\end{abstract}

\section{Introduction}

The term "full abstraction" was coined by Robin Milner in Mil75. In 1977 two seminal papers on the programming language PCF, by Milner and Plotkin, appeared in Theoretical Computer Science [Mil77, Plo77]. These papers initiated an extensive body of work centering on the Full Abstraction problem for PCF (see [BCL86, Cur93, Ong95] for surveys).

The importance of full abstraction for the semantics of programming languages is that it is one of the few quality filters we have. Specifically, it provides a clear criterion for assessing how definitive a semantic analysis of some language is. It must be admitted that to date the quest for fully abstract models has not yielded many obvious applications; but it has generated much of the deepest work in semantics. Perhaps it is early days yet.

Recently, game semantics has been used to give the first syntax-independent constructions of fully abstract models for a number of programming languages, including PCF [AJM96, HO96, Nic94, richer functional languages [AM95, McC96b, McC96a, HY97, and languages with non-functional features such as reference types and non-local control constructs AM97c, AM97b, AM97a, Lai97. A noteworthy feature is that the key definability results for the richer languages are proved by a reduction to definability for the functional fragment, using a technique of factorization theorems. Thus the results originally obtained for PCF prove to be a lynch-pin in the analysis of a much wider class of languages.

When some success has been achieved with concrete constructions, it becomes important to identify the key properties of these constructions at a more abstract level; thus the trend towards axiomatic and synthetic domain theory, for example [Fio96, Hyl91]. There has also been considerable progress in axiomatizing sufficient conditions for computational adequacy [FP94, Bra97, McC96a]. In another vein, the work on action structures [MMP95] can be seen as an axiomatics for process calculi and other computational formalisms.

In the present paper we make the first contribution towards an axiomatic account of full abstraction, with specific reference to PCF. We present axioms on models of PCF from which the key results on definability and full abstraction can be proved. It should be emphasized that not only the results of [AJM96], but also the top-level structure of the actual proofs, are captured by our axiomatic account. In particular, our main axioms are abstracted from 
key lemmas in AJM96]. The axioms mostly take the form of assertions that some canonical map is an isomorphism, which is quite standard in categorical axiomatizations, for example of Synthetic Differential Geometry [Koc81], distributive categories [Wal92], or dualizing objects MOM91]. It is also noteworthy that, although our results apply to intuitionistic types, the axioms make essential use of the linear decompositions of these types [Gir87.

The present paper is only a first step. We hope that it will lead to further work in a number of directions:

- a more abstract perspective on game semantics

- a synthetic/axiomatic account of sequentiality

- general results on full abstraction

- a fine structure theory of "behavioural features" of categorical models.

\section{Preliminaries}

This section is concerned with setting up some basic language in which the substantive axioms can be expressed.

\subsection{Affine categories}

We firstly recall the standard definition of a categorical model for the $(\otimes, \multimap, \times, 1, !)$ fragment of Affine logic [See87, Bie95].

An affine category is a symmetric monoidal closed category $\mathcal{C}$ with finite products, such that the tensor unit is the terminal object 1 , together with a comonad which we write in "co-Kleisli form" Man76] as $\left(!, \operatorname{der},(\cdot)^{\dagger}\right)$ where ! : $\mathcal{C} \rightarrow \mathcal{C}, \operatorname{der}_{A}: ! A \rightarrow A$ for all $A$, and

$$
(\cdot)_{A, B}^{\dagger}: \mathcal{C}(! A, B) \rightarrow \mathcal{C}(! A, ! B)
$$

satisfy:

$$
\begin{aligned}
f^{\dagger} ; g^{\dagger} & =\left(f^{\dagger} ; g\right)^{\dagger} \\
f^{\dagger} ; \operatorname{der} & =f \\
\operatorname{der}^{\dagger} ; f & =f .
\end{aligned}
$$

There are moreover natural isomorphisms (the "exponential laws")

$$
\begin{array}{ccc}
e_{A, B} & : & !(A \times B) \stackrel{\cong}{\cong} ! A \otimes ! B \\
e_{1} & : & ! 1 \stackrel{\cong}{\longrightarrow} 1
\end{array}
$$

satisfying the coherence conditions given in Bie95]. Every object !A has a cocommutative comonoid structure given by

$$
\begin{gathered}
\operatorname{con}_{A}=! A \stackrel{! \Delta}{\longrightarrow} !(A \times A) \stackrel{e_{A, A}}{\longrightarrow} ! A \otimes ! A \\
\operatorname{weak}_{A}=! A \stackrel{! \mathrm{t}_{A}}{\longrightarrow} ! 1 \stackrel{e_{1}}{\longrightarrow} 1 .
\end{gathered}
$$

The co-Kleisli category $K_{!}(\mathcal{C})$ is cartesian closed, with products as in $\mathcal{C}$, and function space

$$
A \Rightarrow B=! A-\circ B .
$$




\subsection{Partiality}

Recall firstly that Set $_{\star}$, the category of pointed sets, has as objects sets with a specified base point, and as morphisms functions preserving the base point. Set $_{\star}$ is symmetric monoidal closed, with tensor given by smash product and function spaces by basepoint-preserving maps. It has products as in Set, and coproducts given by coalesced sums (i.e. disjoint unions with basepoints identified). We write $\amalg$ for disjoint union of sets, and + for coproduct in Set $_{\star}$. We write $X_{\star}$ for an object of Set $_{\star}$, where $X$ is the set of elements excluding the basepoint. Thus $X_{\star}+Y_{\star}=(X \coprod Y)_{\star}$.

Now let $\mathcal{C}$ be a category with finite products. $\mathcal{C}$ has $\perp$-maps if for each object $A$ there is a distinguished morphism $\perp_{A}: 1 \rightarrow A$. We then define $\perp_{A, B}$ for all $A, B$ by

$$
\perp_{A, B}=A \stackrel{\mathrm{t}_{A}}{\longrightarrow} 1 \stackrel{\perp_{B}}{\longrightarrow} B .
$$

We require that $\perp_{B, C} \circ f=\perp_{A, C}$ for all objects $A, B, C$ and $f: A \rightarrow B$; and that

$$
\perp_{A \times B}=\left\langle\perp_{A}, \perp_{B}\right\rangle .
$$

A morphism $f: A \rightarrow B$ is strict if $f \circ \perp_{A}=\perp_{B}$. The strict morphisms form a subcategory which we denote $\mathcal{C}_{s}$. Note that $\mathcal{C}_{s}$ is enriched over Set $_{\star}$. Thus there is an enriched hom-functor

$$
\mathcal{C}_{s}(-,-): \mathcal{C}_{s}^{o} \times \mathcal{C}_{s} \longrightarrow \text { Set }_{\star} .
$$

Note also that for any object $A$,

$$
\mathrm{C}(A,-): \mathrm{C}_{s} \longrightarrow \text { Set }_{\star}
$$

is a well-defined functor, since the basepoint of $\mathcal{C}(A, B)$ is $\perp_{A, B}$, and for any strict $f: B \rightarrow C$,

$$
f \circ \perp_{A, B}=\perp_{A, C} .
$$

A morphism $f: A \rightarrow B$ is total if it is strict and $f \neq \perp_{A, B}$. We write $\mathcal{C}_{t}(A, B)$ for the set of total morphisms from $A$ to $B$, so that

$$
\mathcal{C}_{s}(A, B)=\mathcal{C}_{t}(A, B)_{\star}
$$

However, note that total morphisms need not be closed under composition.

Examples In the category $\mathbf{C p o}$ of directed-complete partial orders with least elements and continuous maps, strictness has its expected meaning.

In the categories of games in [AJM96, HO96, McC96a], the $\perp$-maps are the empty strategies. The strict strategies $\sigma: A \rightarrow B$ are those which respond to the opening move by Opponent (which must be in $B$ ) with a move in $A$ if they have any response at all.

\subsection{Atomic and discrete objects}

Let $\mathcal{C}$ be a category with finite products and $\perp$-maps. An object $B$ of $\mathcal{C}$ is a $\pi$-atom (cf. Joy95a, Joy95b) if

$$
\mathcal{C}_{s}(-, B): \mathfrak{C}_{s}^{\circ} \longrightarrow \text { Set }_{\star}
$$


preserves coproducts, i.e. for each finite family $\left\{A_{i} \mid i \in I\right\}$ of objects in $\mathcal{C}$, the canonical map

$$
\begin{gathered}
\sum_{i \in I} \mathcal{C}_{s}\left(A_{i}, B\right)=\left(\coprod_{i \in I} \mathcal{C}_{t}\left(A_{i}, B\right)\right)_{\star} \longrightarrow \mathcal{C}_{s}\left(\prod_{i \in I} A_{i}, B\right) \\
\left\{\begin{array}{ccc}
(i, f) & \mapsto & \pi_{i} ; f \\
* & \mapsto & \prod_{\prod_{i \in I} A_{i}, B}
\end{array}\right.
\end{gathered}
$$

is a bijection. The motivation for this terminology comes from lattice theory (Joyal is generalizing Whitman's theorem on free lattices). A $\pi$-atom in a lattice (also often called a meet-irreducible element, $c f$. [DP90]) is an element $a$ such that

$$
\bigwedge_{i=1}^{n} a_{i} \leq a \Longrightarrow \exists i . a_{i} \leq a .
$$

Generalizing from posets to (enriched) categories, we get the definition given above.

An object $B$ is discrete if for each $A$ the canonical map

$$
\begin{gathered}
\mathcal{C}_{s}(A, B)+\mathcal{C}(1, B) \longrightarrow \mathcal{C}(A, B) \\
f: A \rightarrow_{s} B \mapsto f: A \rightarrow B, \quad x: 1 \rightarrow B \mapsto A \rightarrow 1 \stackrel{x}{\rightarrow} B
\end{gathered}
$$

is a bijection.

The idea behind this definition is that any morphism into a discrete object is either strict or constant. It should be recalled that the coproduct on the left is the coalesced sum in Set $_{\star}$; this allows the constant $-\perp$ morphism (which is both strict and constant) to be properly accounted for.

We write $\mathcal{C}^{\pi}$ for the full subcategory of $\mathcal{C}$ determined by the $\pi$-atomic objects.

Examples In the (Linear) categories of games in AJM96, HO96, McC96a], any game $B$ with a unique initial question is $\pi$-atomic. The response to this unique initial question in $B$ made by a total strategy $\prod_{i \in I} A_{i} \rightarrow B$ must be in one of the games $A_{i}$. Making such a move entails projecting the product onto the $i$ 'th factor. Flat games-i.e. those with a unique initial question and a set of possible answers, after which nothing more can happen - are discrete. This just says that if $A$ is discrete, then any strategy $A \rightarrow B$ is either the empty strategy, or responds to the unique initial question in $B$ with some move in $A$-and hence is strict; or responds with an answer in $B$ which completes the play, and hence is a "constant" strategy.

In Cpo, flat domains are discrete (any continuous function into a flat domain is either strict or constant); Coh, the category of coherence spaces and linear maps, is soft in the sense of [Joy95a, Joy95b-see [HJ97.

\subsection{Standard datatypes}

Let $\mathcal{C}$ be a category with $\perp$-maps as in Section 2.2. We assume given a class of objects of $\mathcal{C}$ which we will call "well-opened", which forms an exponential ideal, i.e. if $B$ is well-opened os is $A-\circ B$, and which moreover is closed under products. We write $\mathcal{C}^{w o}$ for the sub-category of well-opened objects.

We say that $\mathcal{C}$ has standard datatypes if: 
- The total maps on $\mathcal{C}^{w o}$ form a sub-category $\mathcal{C}_{t}^{w o}$.

- The functor

$$
\mathcal{C}(1,-): \mathcal{C}_{t}^{w o} \longrightarrow \text { Set }_{\star}
$$

has a left adjoint left inverse $\widetilde{(\cdot)}$.

Unpacking this definition, for each well-opened object $A$ of $\mathcal{C}$ and pointed set $X_{\star}$, there is an isomorphism

$$
\mathcal{C}_{t}^{w o}\left(\widetilde{X_{\star}}, A\right) \cong \operatorname{Set}_{\star}\left(X_{\star}, \mathcal{C}(1, A)\right)
$$

natural in $X_{\star}$ and $A$, such that the unit

$$
\eta_{X_{\star}}: X_{\star} \longrightarrow \mathcal{C}\left(1, \widetilde{X_{\star}}\right)
$$

is an isomorphism. In particular, $X_{\star}$ is well-opened, and there are maps

$$
\bar{x}: 1 \rightarrow \widetilde{X_{\star}} \quad(x \in X)
$$

with $\bar{x} \neq \perp_{\widetilde{X_{\star}}}$, and for each family

$$
\left(f_{x}: 1 \rightarrow A \mid x \in X\right)
$$

a unique total morphism

$$
\left[f_{x} \mid x \in X\right]: \widetilde{X_{\star}} \rightarrow A
$$

such that

$$
\bar{x}_{0} ;\left[f_{x} \mid x \in X\right]=f_{x_{0}} \quad\left(x_{0} \in X\right) .
$$

Examples In the categories of games in AJM96, HO96, McC96a, $\widetilde{X}_{\star}$ is the "flat game" with a unique initial question, and one answer for each $x \in X$. The well-opened objects in these categories are those in which any move that can occur as the first move in a play can only so occur.

In Cpo, a slightly different situation prevails. The functor

$$
\mathbf{C p o}(1,-): \mathbf{C p o}_{s} \longrightarrow \mathbf{S e t}_{\star}
$$

has a left adjoint left inverse, which sends $X_{\star}$ to the flat domain $X_{\perp}$.

\section{Sequential Categories}

Let $\mathcal{C}$ be an affine category with $\perp$-maps and standard datatypes. $\mathcal{C}$ is a sequential category if it satisfies the following axioms (A1)-(A5).

(A1) $\widetilde{X}_{\star}$ is discrete for each set $X$.

(A2) $\widetilde{X}_{\star}$ is $\pi$-atomic for each set $X$, and $\mathcal{C}^{\pi}$ is an exponential ideal. 
(A3) (Uniformity of threads). Let $A$ and $B$ be well-opened objects. Then

$$
\mathcal{C}(! A, ! B) \cong \mathcal{C}(! A, B) \text {. }
$$

More precisely, there are canonical maps

$$
\begin{array}{llcll}
f: ! A \rightarrow ! B & \mapsto & f ; \operatorname{der}_{B} & : & ! A \rightarrow B \\
g: ! A \rightarrow B & \mapsto & g^{\dagger} & : & ! A \rightarrow ! B
\end{array}
$$

and since $\left(!\right.$, der,$\left.(\cdot)^{\dagger}\right)$ is a comonad,

$$
g^{\dagger} ; \operatorname{der}_{B}=g
$$

The content of (A3) is that

$$
\left(f ; \operatorname{der}_{B}\right)^{\dagger}=f
$$

i.e. that the two passages are mutually inverse.

This property was proved for categories of games in [AJM96] and subsequently in [McC96a, under the name of the "Bang Lemma". The idea is that morphisms $f: ! A \rightarrow ! B$ must display uniform behaviour in all "threads", i.e. in each copy of $B$ together with its associated copies of $A$. This property holds in these categories as a consequence of history-freeness in [AJM96], and of innocence in $\mathrm{McC96a}$. The idea in each case is that a strategy $f: ! A \rightarrow ! B$ can only "see" the current thread, and hence must play like the promotion of its restriction to a single thread, i.e. like $\left(f ; \operatorname{der}_{B}\right)^{\dagger}$.

\section{(A4) (Linearization of Head Occurrence).}

$$
\mathcal{C}_{s}(! A, B) \cong \mathcal{C}_{s}(A, ! A-\circ B) .
$$

More precisely, there is a canonical map

$$
\begin{aligned}
\mathcal{C}_{s}(A, ! A-\circ B) & \\
& \mid \Lambda^{-1} \\
\mathcal{C}(A \otimes ! & A, B) \\
& \mid \mathrm{C}\left(\operatorname{der}_{A} \otimes \mathrm{id}_{! A}, \mathrm{id}_{B}\right) \\
\mathrm{C}(! A \otimes ! A, B) & ! \\
& \mid \mathrm{C}\left(\operatorname{con}_{A}, \mathrm{id}_{B}\right) \\
\mathrm{C}(! A, B) &
\end{aligned}
$$

The content of (A4) is firstly that this map factors through the inclusion

$$
\mathcal{C}_{s}(! A, B) \hookrightarrow \mathcal{C}(! A, B)
$$

and secondly that the corestriction

$$
\mathcal{C}_{s}(A, ! A-\circ B) \longrightarrow \mathcal{C}_{s}(! A, B)
$$

is a bijection.

This property was proved, under the name of "Separation of Head Occurrence", for categories of games firstly in [AJM96], and subsequently in [McC96a. There is a suggestive analogy, at least, with operational and proof-theoretic ideas of treating head variables linearly Gir87, DHR96]. The idea is simply that we can split the use of many copies of $A$ in ! $A$ on the left into the tensor product of the first copy used, of type $A$, and the remaining copies, of type $! A$. In the case of strict strategies, which are either empty or use at least one copy of $A$, this correspondence is biunique. 
(A5) (Linear Function Extensionality). There is an isomorphism

$$
\mathcal{C}_{s}(A-\circ B, ! C-\circ D) \cong \mathcal{C}(! C, A)_{\star} \otimes \mathcal{C}_{s}(B, ! C-\circ D)
$$

provided $B$ and $D$ are discrete. (The tensor product on the right is smash product in Set $_{\star}$. Note that

$$
\left.\mathcal{C}(! C, A)_{\star} \otimes \mathcal{C}_{s}(B, ! C-\circ D)=\left(\mathcal{C}(! C, A) \times \mathcal{C}_{t}(B, ! C-\circ D)\right)_{\star} \cdot\right)
$$

More precisely, there is a canonical map

$$
\begin{aligned}
& \mathcal{C}(! C, A) \times \mathcal{C}_{t}(B, ! C \multimap D) \\
& \downarrow(-)-\circ(-) \\
& \mathcal{C}(A \multimap B, ! C \multimap(! C \multimap D)) \\
& \downarrow \cong \\
& \mathcal{C}(A \multimap B, ! C \otimes ! C \multimap D) \\
& \downarrow \mathfrak{e}\left(\text { id, } \operatorname{con}_{C}-\mathrm{oid}\right) \\
& \mathcal{C}(A-\circ B, ! C-\circ D)
\end{aligned}
$$

The content of (A5) is that this map factors through the inclusion

$$
\mathcal{C}_{t}(A-\circ, ! C-\circ) \hookrightarrow \mathcal{C}(A-\circ B, ! C-\circ D)
$$

and that the corestriction

$$
\mathcal{C}(! C, A) \times \mathcal{C}_{t}(B, ! C-\circ D) \longrightarrow \mathcal{C}_{t}(A-\circ B, ! C-\circ D)
$$

and hence also the "lifted" map

$$
\mathcal{C}(! C, A)_{\star} \otimes \mathcal{C}_{s}(B, ! C-\circ D) \longrightarrow \mathcal{C}_{s}(A-\circ B, ! C-\circ D)
$$

is a bijection.

A special case of this property, under the same name, was proved for categories of games in AJM96], and subsequently in [McC96a. The general case was implicit in the proof of the Decomposition Theorem in AJM96. Intuitively, this axiom says that the only thing we can do with a linear functional parameter is to apply it to an argument, and apply some function to the result. The verification of this axiom in the categories of games considered in AJM96, McC96a makes essential use both of history-freeness/innocence, and of wellbracketedness. The idea is that a strict strategy of the type displayed in the axiom must respond to the initial question in $D$ by "calling its function argument", i.e. by making the (unique) initital move in $B$. By the bracketing condition, the initial question in $D$ cannot be answered until the initial question in $B$ has been answered, i.e. until the play in $B$ is complete. This allows us to decompose the strategy we started with into sub-strategies, corresponding to what is done before and after the play in $B$ is completed. History-freeness/innocence then guarantess that the continuation strategy which proceeds after the play in $B$ is completed can depend only on the answer returned, not on the interaction which took place between the function and its arguments. This is one of the key points where essentially non-functional behaviour is being excluded. 
Examples A minor embarassment is that neither of our two concrete examples of models for the above axioms, namely the categories of games described in [AJM96, McC96a, quite succeeds in being a sequential category! They do satisfy the key axioms (A1)-(A5). In the case of the category in [AJM96, the problem is that it fails to have products in the underlying Affine category - although the co-Kleisli category is cartesian closed. However, there is a "candidate" for the product in the Affine category - which turns into a real product in the co-Kleisli category - which does have projections, and with respect to which the required properties relating to $\pi$-atomicity do hold. This is enough for our applications to definability to follow. Similarly, in the category used in McC96a ! fails to be a co-monad; however, one does get a cartesian closed co-Kleisli category by restricting to the well-opened objects.

These minor mismatches are probably best taken as an indication that we do not yet have a definitive formulation of game semantics.

\section{Decomposition}

We will now prove a decomposition theorem in the sense of AJM96 from the axioms.

Let $\mathcal{C}$ be a sequential category. Let

$$
A_{i}=!\left(B_{i, 1} \times \cdots \times B_{i, q_{i}}\right)-\circ \widetilde{X_{\star}} \quad(1 \leq i \leq k)
$$

be objects of $\mathcal{C}$. We write $\vec{A}=A_{1} \times \cdots \times A_{k}$. Consider a morphism $f: ! \vec{A} \rightarrow \widetilde{X}_{\star}$. By (A1), $\widetilde{X_{\star}}$ is discrete, hence three disjoint cases apply:

- $f=\perp$.

- $f=! \vec{A} \rightarrow 1 \stackrel{g}{\rightarrow} \widetilde{X_{\star}}$. In this case, by the universal property of $\widetilde{X_{\star}}$, we must have $g=\bar{x}$ for some unique $x \in X$.

- $f$ is total. In this case, by (A4) $f$ is in bijective correspondence with a total morphism

$$
f^{\prime}: \vec{A} \rightarrow ! \vec{A}-\circ \widetilde{X_{\star}} .
$$

By (A2), $f^{\prime}$ factors by a projection $\pi_{i}$ through

$$
f_{i}: A_{i} \rightarrow_{t} ! \vec{A}-\circ \widetilde{X_{\star}}
$$

for a unique $i, 1 \leq i \leq k$. By (A5), $f_{i}$ decomposes into

$$
g: ! \vec{A} \rightarrow ! \vec{B}_{i}, \quad h: \widetilde{X_{\star}} \rightarrow_{t}\left(! \vec{A}-\circ \widetilde{X_{\star}}\right)
$$

where $\vec{B}_{i}=B_{i, 1} \times \cdots \times B_{i, q_{i}}$. By (A3), $g=\left(g \text {; der } \vec{B}_{i}\right)^{\dagger}$, and by the universal property of the product,

$$
g=\left\langle g_{1}, \ldots, g_{q_{i}}\right\rangle^{\dagger}
$$

for unique $g_{j}: ! \vec{A} \rightarrow B_{i, j}, 1 \leq j \leq q_{i}$. By the universal property of $\widetilde{X_{\star}}$,

$$
h=\left[h_{x}: 1 \rightarrow ! \vec{A}-\circ \widetilde{X}_{\star} \mid x \in X\right] .
$$

Thus we obtain

$$
f=\mathbf{C}_{i}\left(g_{1}, \ldots, g_{q_{i}},\left(h_{x} \mid x \in X\right)\right)
$$

where $\mathbf{C}_{i}\left(g_{1}, \ldots, g_{q_{i}},\left(h_{x} \mid x \in X\right)\right)$ abbreviates

$$
\operatorname{con}_{\vec{A}} ;\left(\operatorname{con}_{\vec{A}} ;\left(\operatorname{der}_{\vec{A}} ; \pi_{i}\right) \otimes\left\langle g_{1}, \ldots, g_{q_{i}}\right\rangle^{\dagger} ; \mathrm{Ap}\right) \otimes \operatorname{id}_{! \vec{A}} ; \Lambda^{-1}\left(\left[h_{x} \mid x \in X\right]\right) .
$$


We summarize this analysis in

Theorem 4.1 (Decomposition) With notation as above, one of the following three cases applies:

- $f=\perp$.

- $f=! \vec{A} \rightarrow 1 \stackrel{\bar{x}}{\rightarrow} \widetilde{X_{\star}}$.

- $f=\mathbf{C}_{i}\left(g_{1}, \ldots, g_{q_{i}},\left(h_{x} \mid x \in X\right)\right)$.

Moreover, this decomposition is unique.

\section{$5 \quad \mathrm{PCF}$}

In this section we briefly recall the language PCF, its operational semantics and observational preorder, in a streamlined version convenient for our purposes.

PCF is an applied simply typed $\lambda$-calculus with a single base type nat. The constants are as follows:

- recursion combinators $\mathbf{Y}_{T}:(T \Rightarrow T) \Rightarrow T$ for each type $T$.

- $\Omega$ : nat.

- $\underline{n}$ : nat for each $n \in \mathbb{N}$.

- case $_{k}$ : nat $\Rightarrow \underbrace{\text { nat } \Rightarrow \cdots \Rightarrow \text { nat }}_{k} \Rightarrow$ nat for each $k \in \mathbb{N}$.

The main difference from PCF as originally presented by Scott and Plotkin is in the use of the case $_{k}$ constants instead of the more familiar conditionals and arithmetic operations. The case $_{k}$ constants are needed for a precise correspondence at the intensional level, and as shown in detail in AJM96, the difference is insignificant as far as observational equivalence is concerned.

The operational semantics is defined via a structural congruence $\equiv(c f$. Mil92] $)$ and an evaluation relation _ $\Downarrow_{-}$. The structural congruence is the congruence on terms generated by $\beta \eta$-conversion and all instances of

$$
\mathbf{Y} M \equiv M(\mathbf{Y} M) .
$$

The evaluation relation $P \Downarrow \underline{n}$ is defined between programs, i.e. closed terms of type nat, and numerals $\underline{n}$, inductively as follows:

$$
\begin{gathered}
\frac{M \equiv M^{\prime} \quad M^{\prime} \Downarrow \underline{n}}{M \Downarrow \underline{n}} \quad \underline{\underline{n} \Downarrow \underline{n}} \\
P \Downarrow \underline{i}(i<k) \quad P_{i} \Downarrow \underline{n} \\
\operatorname{case}_{k} P P_{0} \cdots P_{k-1} \Downarrow \underline{n}
\end{gathered}
$$

Let $\operatorname{Trm}(\Gamma, T)$ be the set of terms $M$ such that $\Gamma \vdash M: T$ is derivable. Let $\operatorname{Ctxt}(\Gamma, T)$ be the set of contexts $C[\cdot]$ such that $C[M]$ is a program for all $M \in \operatorname{Trm}(\Gamma, T)$. The observational preorder is defined at $\Gamma, T$ by:

$$
M \underset{\sim}{\sqsubset}, T=\forall C[\cdot] \in \operatorname{Ctxt}(\Gamma, T) . C[M] \Downarrow \underline{n} \Longrightarrow C[N] \Downarrow \underline{n} .
$$




\section{Computational Adequacy}

Let $\mathcal{C}$ be an affine category with $\perp$-maps and standard datatypes. The cartesian closed category $K_{!}(\mathrm{C})$ provides a model of the fragment of PCF obtained by omitting the recursion combinators $\mathbf{Y}_{T}$. The base type nat is interpreted by $\widetilde{\mathbb{N}}_{\star}$, the constants $\underline{n}$ by $\bar{n}, n \in \mathbb{N}$, and $\Omega$ by $\perp_{\widetilde{\mathbb{N}_{\star}}}$. The constant case in interpreted by

$$
\operatorname{der}_{\widetilde{N}_{\star}} ;\left[f_{i} \mid i \in \mathbb{N}\right]
$$

where

$$
f_{i}= \begin{cases}\Lambda^{k}\left(\pi_{i}\right), & 0 \leq i<k \\ \perp, & i \geq k .\end{cases}
$$

This interpretation is extended to all terms in the standard way [Cro94].

To accommodate recursion, we need another definition. Let $\mathcal{K}$ be a cartesian closed category. A fixpoint operator on $\mathcal{K}$ is a family of maps

$$
()_{A}^{\nabla}: \mathcal{K}(A, A) \longrightarrow \mathcal{K}(1, A)
$$

satisfying

$$
f \circ f^{\nabla}=f^{\nabla} .
$$
by

Given such an operator, we can interpret the fixpoint combinator $\mathbf{Y}_{A}: 1 \rightarrow(A \Rightarrow A) \Rightarrow A$

$$
\llbracket F:(A \Rightarrow A) \Rightarrow A \vdash \lambda f: A \Rightarrow A . f(F f) \rrbracket^{\nabla} .
$$

A model is said to be computationally adequate if, for all programs $P$ and $n \in \mathbb{N}$ :

$$
P \Downarrow \underline{n} \Longleftrightarrow \llbracket P \rrbracket=\bar{n} .
$$

Let $\mathcal{C}$ be an affine category with $\perp$-maps and standard datatypes, equipped with a fixpoint operator on $K_{!}(\mathcal{C})$. $\mathcal{C}$ is said to be continuously observable if, for all $f: A \rightarrow A$ and $g: A \rightarrow \widetilde{X}_{\star}$ in $K_{!}(\mathcal{C})$, and all $x \in X$ :

$$
g \circ f^{\nabla}=\bar{x} \Longleftrightarrow \exists k \in \omega \cdot g \circ f^{k} \circ \perp=\bar{x} .
$$

Recall from AJM96] that a rational cartesian closed category is a cartesian closed category enriched over pointed posets, with least upper bounds of "definable" chains, i.e. those of the form $\left(f^{k} \circ \perp \mid k \in \omega\right)$. This provides just enough structure to interpret the recursion combinators $\mathbf{Y}$ in terms of least fixpoints. It is shown in AJM96 that the category of games studied there is rational; this, together with the fact that the "points" of the standard datatypes form flat domains implies that the category is continuously observable.

Theorem 6.1 (Computational Adequacy) If $\mathcal{C}$ is continuously observable then $K_{!}(\mathcal{C})$ is a computationally adequate model of PCF.

The original proof by Plotkin for the Scott continuous function model [Plo77] goes through in our axiomatic setting ( $c f$. [Bra97]).

In practice, it is often more convenient to verify somewhat stronger axioms. We say that a sequential category $\mathcal{C}$ is normed if:

- It is enriched over algebraic cpo's, with the $\perp$-morphisms being the least elements in the partial orderings, and such that $\mathcal{C}\left(1, \widetilde{X_{\star}}\right)$ is order-isomorphic to the flat domain $X_{\perp}$. 
- There is a norm function $\|f\| \in \mathbb{N}$ on compact morphisms $f$, such that, for each PCF type $T$ and compact $f: 1 \longrightarrow \llbracket T \rrbracket$ :

$$
\begin{gathered}
f=\mathbf{C}_{i}\left(f_{1}, \ldots, f_{q_{i}},\left(g_{n} \mid n \in \mathbb{N}\right)\right) \Longrightarrow \\
\sup \left(\left\{\left\|f_{j}\right\| \mid 1 \leq j \leq q_{i}\right\} \cup\left\{\left\|g_{n}\right\| \mid n \in \mathbb{N}\right\}\right)<\|f\|,
\end{gathered}
$$

and $g_{n}=\perp$ for almost all $n \in \mathbb{N}$.

Note that a normed category is automatically observably continuous and hence computationally adequate.

The categories in [HO96, McC96a] are normed, with the norm of a compact innocent strategy given by the cardinality of its view function.

\section{Definability}

Let $T=T_{1} \Rightarrow \cdots T_{k} \Rightarrow$ nat be a PCF type. Note that

$$
\begin{aligned}
\llbracket T \rrbracket & =\llbracket T_{1} \rrbracket \Rightarrow \cdots \llbracket T_{k} \rrbracket \Rightarrow \llbracket \text { nat } \rrbracket \\
& =! \llbracket T_{1} \rrbracket-\circ \cdots ! \llbracket T_{k} \rrbracket-\circ \widetilde{\mathbb{N}}_{\star} \\
& \cong\left(! \llbracket T_{1} \rrbracket \otimes \cdots \otimes ! \llbracket T_{k} \rrbracket\right)-\circ \widetilde{\mathbb{N}}_{\star} \\
& \cong !\left(\llbracket T_{1} \rrbracket \times \cdots \times \llbracket T_{k} \rrbracket\right)-\circ \widetilde{\mathbb{N}}_{\star} .
\end{aligned}
$$

To save notational overhead, we shall elide this canonical isomorphism, equating the "curried" and "uncurried" versions of types. (However, for a careful treatment in which these isomorphisms are made explicit, see [AJM96]).

Let $\mathcal{C}$ be a sequential category. For each $f: 1 \rightarrow \llbracket T \rrbracket$ in $\mathcal{C}$ and $k \in \omega$, we define $p_{k}(f)$ inductively as follows:

$$
\begin{aligned}
& p_{0}(f)=\perp \\
& p_{k+1}(f)= \begin{cases}\perp, & f=\perp \\
f, & f=\mathrm{t} ; \bar{n} \\
f^{\prime}, & f=\mathbf{C}_{i}\left(f_{1}, \ldots, f_{q_{i}},\left(g_{n} \mid n \in \mathbb{N}\right)\right)\end{cases}
\end{aligned}
$$

where

$$
\begin{aligned}
& f^{\prime}=\mathbf{C}_{i}\left(p_{k}\left(f_{i}\right), \ldots p_{k}\left(f_{q_{i}}\right),\left(h_{n} \mid n \in \mathbb{N}\right)\right) \\
& h_{n}= \begin{cases}p_{k}\left(g_{n}\right), & 0 \leq n<k \\
\perp & n \geq k .\end{cases}
\end{aligned}
$$

This definition by cases is valid by the Decomposition Theorem.

Theorem 7.1 (Definability) For each PCF type T, $f: 1 \rightarrow \llbracket T \rrbracket$ and $k \in \omega, p_{k}(f)$ is definable in PCF. That is, there exists a PCF term $\vdash M: T$ such that $p_{k}(f)=\llbracket M \rrbracket$.

Proof By induction on $k$, and cases on the decomposition of $f$. We write $T=\widetilde{T} \Rightarrow$ nat, where $\widetilde{T}=T_{1}, \ldots, T_{k}$.

$$
\begin{array}{ll}
p_{0}(f) & =\quad \perp=\llbracket \lambda \tilde{x}: \widetilde{T} \cdot \Omega \rrbracket \\
p_{k+1}(\perp) & =\quad \perp=\llbracket \lambda \tilde{x}: \widetilde{T} \cdot \Omega \rrbracket \\
p_{k+1}(\mathrm{t} ; \bar{n}) & =\mathrm{t} ; \bar{n}=\llbracket \lambda \tilde{x}: \widetilde{T} \cdot \underline{n} \rrbracket
\end{array}
$$




$$
\begin{gathered}
p_{k+1}\left(\mathbf{C}_{i}\left(p_{k}\left(f_{i}\right), \ldots p_{k}\left(f_{q_{i}}\right),\left(h_{n} \mid n \in \mathbb{N}\right)\right)\right)= \\
\llbracket \lambda \tilde{x}: \widetilde{T} . \operatorname{case}_{k}\left(x_{i}\left(M_{1} \tilde{x}\right) \cdots\left(M_{q_{i}} \tilde{x}\right)\right)\left(P_{0} \tilde{x}\right) \cdots\left(P_{k-1} \tilde{x}\right) \rrbracket
\end{gathered}
$$

where

$$
\begin{aligned}
& p_{k}\left(f_{j}\right)=\llbracket M_{j} \rrbracket, \quad 1 \leq j \leq q_{i}, \\
& p_{k}\left(g_{n}\right)=\llbracket P_{n} \rrbracket, \quad 0 \leq n<k .
\end{aligned}
$$

For normed categories, we can prove a stronger result.

Theorem 7.2 (Definability for Normed Sequential Categories) For each PCF type T and compact $f: 1 \rightarrow \llbracket T \rrbracket, f$ is definable in PCF. That is, there exists a PCF term $\vdash M: T$ such that $f=\llbracket M \rrbracket$.

Proof By complete induction on $\|f\|$, and cases on the decomposition of $f$. We write $T=\widetilde{T} \Rightarrow$ nat, where $\widetilde{T}=T_{1}, \ldots, T_{k}$. If $f=\perp$, then

$$
f=\llbracket \lambda \tilde{x}: \tilde{T} \cdot \Omega \rrbracket .
$$

If $f=\mathrm{t} ; \bar{n}$, then

$$
f=\llbracket \lambda \tilde{x}: \widetilde{T} \cdot \underline{n} \rrbracket .
$$

If $f=\mathbf{C}_{i}\left(f_{1}, \ldots, f_{q_{i}},\left(g_{n} \mid n \in \mathbb{N}\right)\right)$, then

$$
f=\llbracket \lambda \tilde{x}: \widetilde{T} \cdot \operatorname{case}_{l}\left(x_{i}\left(M_{1} \tilde{x}\right) \cdots\left(M_{q_{i}} \tilde{x}\right)\right)\left(P_{0} \tilde{x}\right) \cdots\left(P_{k-1} \tilde{x}\right) \rrbracket
$$

where by induction hypothesis

$$
\begin{aligned}
f_{j} & =\llbracket M_{j} \rrbracket, \quad 1 \leq j \leq q_{i}, \\
g_{n} & =\llbracket P_{n} \rrbracket, \quad 0 \leq n<l,
\end{aligned}
$$

and $g_{m}=\perp$ for all $m \geq l$.

We note that the terms used to exhibit definability in Theorems 7.1 and 7.2 are of a special form; the evaluation trees of [AJM96. These can be seen as a form of Bohm tree appropriate for PCF. These trees were in fact first identified as the right notion of normal forms for PCF terms as a consequence of the work on game semantics; this is an interesting example of feedback from semantics to syntax. In AJM96] it is shown that there is an order-isomorphism between the (possibly infinite) evaluation trees at any PCF type, and the strategies for the game denoted by that type. A similar result can be obtained at the axiomatic level of this paper. Since it is not needed in order to obtain the full abstraction results, we will omit the details.

\section{Full Abstraction}

Let $\mathcal{C}$ be a poset-enriched model of PCF. $\mathcal{C}$ is sound if, for all $M, N \in \operatorname{Trm}(\Gamma, T)$,

$$
\llbracket \Gamma \vdash M: T \rrbracket \leqslant \llbracket \Gamma \vdash N: T \rrbracket \Longrightarrow M \varlimsup_{\Gamma, T} N
$$

and complete if the converse holds. $\mathcal{C}$ is fully abstract if it is both sound and complete.

Lemma 8.1 $\mathrm{C}$ is fully abstract iff soundness and completeness hold for all closed terms. 
Proof The left-to-right implication is immediate. For the converse, we show firstly that, if $\Gamma=x_{1}: T_{k}, \ldots, x_{k}: T_{k}$,

$$
M \varlimsup_{\Gamma, T} N \Longleftrightarrow \lambda \tilde{x}: \tilde{T} \cdot M \varlimsup_{\varnothing, T} \lambda \tilde{x}: \tilde{T} \cdot N .
$$

Again, the left-to-right implication is immediate. For the converse, assume that $\lambda \tilde{x}: \tilde{T} . M \sqsubseteq \Gamma, T$ $\lambda \tilde{x}: \tilde{T} . N$ and that $C[M] \Downarrow \underline{n}$. Define a new context

$$
D[\cdot]=C[[\cdot] \tilde{x}] .
$$

Then $D[\lambda \tilde{x}: \tilde{T} . M] \equiv C[M]$ and hence $D[\lambda \tilde{x}: \tilde{T} . M] \Downarrow \underline{n}$. This implies that $D[\lambda \tilde{x}: \tilde{T} . N] \Downarrow \underline{n}$, but since $D[\lambda \tilde{x}: \tilde{T} . N] \equiv C[N]$, we conclude that $C[N] \Downarrow \underline{n}$, as required.

Furthermore since currying $\Lambda_{A, B, C}: \mathfrak{C}(A \times B, C) \longrightarrow \mathrm{C}(A, B \Rightarrow C)$ is an order-isomorphism, it is immediate that

$$
\llbracket \Gamma \vdash M: T \rrbracket \leqslant \llbracket \Gamma \vdash N: T \rrbracket \Longleftrightarrow \llbracket \vdash \lambda \tilde{x}: \tilde{T} . M \rrbracket \leqslant \llbracket \vdash \lambda \tilde{x}: \tilde{T} . N \rrbracket .
$$

By assumption,

$$
\lambda \tilde{x}: \tilde{T} \cdot M \varpi_{\tilde{T} \Rightarrow T} \lambda \tilde{x}: \tilde{T} \cdot N \Longleftrightarrow \llbracket \lambda \tilde{x}: \tilde{T} \cdot M \rrbracket \leqslant \llbracket \lambda \tilde{x}: \tilde{T} \cdot N \rrbracket .
$$

Combining (44), (5) and (6) we conclude that $\mathcal{C}$ is fully abstract.

Now let $\mathcal{C}$ be an observably continuous sequential category. $K_{!}(\mathcal{C})$ is a computationally adequate model of PCF, by the Computational Adequacy Theorem. We define the intrinsic preorder $\lesssim_{A}$ on each $K_{!}(\mathrm{C})(1, A)$ by

$$
f \lesssim_{A} g \Longleftrightarrow \forall \alpha: A \rightarrow \widetilde{\mathbb{N}}_{\star} \cdot \alpha \circ f=\bar{n} \Rightarrow \alpha \circ g=\bar{n} .
$$

This is extended to general homsets $K_{!}(\mathcal{C})(A, B)$ via the names of morphisms:

$$
f \lesssim_{A, B} g \Longleftrightarrow\ulcorner f\urcorner \lesssim_{A \Rightarrow B}\ulcorner g\urcorner .
$$

Proposition 8.1 For all $A, B \lesssim_{A, B}$ is a preorder, with least element $\perp_{A, B}$, and $K_{!}(\mathrm{C})$ is an enriched cartesian closed category with respect to this preorder. The poset reflection $K_{!}(\mathrm{C}) / \lesssim$ is a rational cartesian closed category, and there is a full cartesian closed functor $Q: K_{!}(\mathcal{C}) \rightarrow K_{!}(\mathcal{C}) / \lesssim$ with the evident universal property which translates the interpretation of PCF in $K_{!}(\mathcal{C})$ to that in $K_{!}(\mathcal{C}) / \lesssim$.

Proof See [HO96, McC96a, Bra97].

We say that $\mathcal{C}$ is approximating if for all PCF types $T, f: \llbracket T \rrbracket \rightarrow \widetilde{X_{\star}}$ and $\alpha: 1 \rightarrow \llbracket T \rrbracket$ in $K_{!}(\mathrm{C})$, and $x \in X$ :

$$
f \circ \alpha=\bar{x} \Longleftrightarrow \exists k \in \omega \cdot p_{k}(f) \circ \alpha=\bar{x} .
$$

In AJM96] it is proved that the category of games considered there is approximating in Section 3.4.

Theorem 8.1 Let $\mathcal{C}$ be an approximating sequential category. Then $K_{!}(\mathcal{C}) / \lesssim$ is a fully abstract model of PCF. 
Proof By the Lemma, it suffices to show soundness and completeness for closed terms. Note that if $M$ is closed, $C[M] \equiv(\lambda x . C[x]) M(x \notin F V(M))$, and hence $C[M] \Downarrow \underline{n} \Longleftrightarrow$ $D[M] \Downarrow \underline{n}$, where $D[\cdot]=(\lambda x . C[x])[\cdot]$. Thus $T$-contexts reduce to applications of functions of type $T \Rightarrow$ nat.

Suppose then that $\llbracket M \rrbracket \leqslant \llbracket N \rrbracket$, and that $C[M] \Downarrow \underline{n}$. By computational adequacy, $\llbracket C[M] \rrbracket=$ $\bar{n}$, i.e. $f \circ \llbracket M \rrbracket=\bar{n}$, where $f=\llbracket \lambda x . C[x] \rrbracket$. This implies that $f \circ \llbracket N \rrbracket=\bar{n}$ i.e. $\llbracket C[N] \rrbracket=\bar{n}$, and by computational adequacy again, $C[N] \Downarrow \underline{n}$. This establishes soundness.

For completeness, suppose that $\llbracket \vdash M: T \rrbracket \nless \llbracket \vdash N: T \rrbracket$, i.e. for some $f: \llbracket T \rrbracket \rightarrow \widetilde{\mathbb{N}}_{\star}$, $f \circ \llbracket M \rrbracket=\bar{n}$ and $f \circ \llbracket N \rrbracket \neq \bar{n}$. Since $\mathcal{C}$ is approximating, for some $k \in \omega, p_{k}(f) \circ \llbracket M \rrbracket=\bar{n}$ and $p_{k}(f) \circ \llbracket N \rrbracket \neq \bar{n}$. By the Definability Theorem, for some $P, p_{k}(f)=\llbracket P \rrbracket$ and hence, defining $C[\cdot]=P[\cdot], \llbracket C[M] \rrbracket=p_{k}(f) \circ \llbracket M \rrbracket=\bar{n}$ while $\llbracket C[N] \rrbracket=p_{k}(f) \circ \llbracket N \rrbracket \neq \bar{n}$. By computational adequacy, $C[M] \Downarrow \underline{n}$ while $\neg(C[N] \Downarrow \underline{n})$, and hence $\neg\left(M \check{\varpi}_{T} N\right)$, as required.

We have the corresponding result for normed sequential categories.

Theorem 8.2 Let $\mathcal{C}$ be a normed sequential category. Then $K_{!}(\mathcal{C}) / \lesssim i s$ a fully abstract model of $P C F$.

Proof The proof is almost identical to that of the preceding theorem. The only difference is in the argument for completeness. Given the separating morphism $f: \llbracket T \rrbracket \rightarrow \widetilde{\mathbb{N}}_{\star}$, we use the compactness of $\bar{n}$ in $\mathcal{C}\left(1, \widetilde{\mathbb{N}}_{\star}\right)$, the algebraicity of $\mathcal{C}\left(\llbracket T \rrbracket, \widetilde{\mathbb{N}_{\star}}\right)$, and the continuity of composition to obtain a compact $f_{0}: \llbracket T \rrbracket \rightarrow \widetilde{\mathbb{N}}_{\star}$ such that $f_{0} \circ \llbracket M \rrbracket=\bar{n}$ and $f_{0} \circ \llbracket N \rrbracket \neq \bar{n}$. Then we use the Definability Theorem for normed sequential categories to obtain the separating context $C[\cdot]$.

\section{$9 \quad$ Full Completeness}

Just as PCF is prototypical for higher-order programming languages, so is the pure simply typed $\lambda$-calculus for logical systems. (Definability results for game semantics of the pure calculus are discussed in [DHR96], and were already known to the authors of [AJM96, HO96].)

We shall indicate how our axiomatic approach can be modified (in fact: simplified) to deal with the pure calculus.

We define a pure sequential category to be an affine category $\mathcal{C}$ with a specified subcategory $\mathcal{C}_{s}$, with the same objects as $\mathcal{C}$, which has an initial object $\iota$ (initial in $\mathcal{C}_{s}$ ), satisfying the following axioms:

(a1) $\mathcal{C}(A, \iota)=\mathcal{C}_{s}(A, \iota)$ for all $A$.

(a2) $\iota$ is $\pi$-atomic (meaning that $\mathcal{C}_{s}(-, \iota): \mathcal{C}_{s}^{\circ} \longrightarrow$ Set preserves finite coproducts), and the $\pi$-atomic objects form an exponential ideal.

(a3) Uniformity of Threads. As in Section 3.

(a4) Linearization of Head Occurrence. As in Section 3. 


\section{(a5) Linear Function Extensionality.}

$$
\mathcal{C}_{s}(A-\circ \iota, ! C-\circ \iota) \cong \mathfrak{C}(! C, A) .
$$

More precisely, the canonical map

$$
(-)-\circ \mathrm{id}_{\iota}: \mathrm{C}(! C, A) \longrightarrow \mathrm{C}(A-\circ \iota, ! C-\circ \iota)
$$

is asserted to corestrict bijectively onto $\mathcal{C}_{s}(A-\circ \iota, ! C-\circ \iota)$. Note by the way that, since $\iota$ is initial in $\mathcal{C}_{s}, \mathcal{C}_{s}(\iota, \iota)=\left\{\mathrm{id}_{\iota}\right\}$

The following decomposition theorem can then be proved for any

$$
f: !\left(\prod_{i \in I} A_{i}\right) \longrightarrow \iota
$$

where

$$
A_{i}=!\left(\prod_{j=1}^{q_{i}} B_{i, j}\right)-\circ \iota, \quad 1 \leq i \leq k
$$

\section{Theorem 9.1 (Decomposition)}

$$
\begin{aligned}
f & =\mathbf{C}_{i}\left(g_{1}, \ldots g_{q_{i}}\right) \\
& \triangleq \operatorname{con}_{\vec{A}} ;\left(\operatorname{der}_{\vec{A}} ; \pi_{i}\right) \otimes\left\langle g_{1}, \ldots, g_{q_{i}}\right\rangle^{\dagger} ; \mathrm{Ap}
\end{aligned}
$$

for a unique $i, 1 \leq i \leq k$, and

$$
f_{j}: !\left(\prod_{i=1}^{k} A_{i}\right) \rightarrow B_{i, j}, \quad 1 \leq j \leq q_{i} .
$$

Proof By (a1), $f$ is strict. By (a4) we obtain a strict morphism $f^{\prime}: \vec{A} \rightarrow(\vec{A}-\circ \iota)$, and by (a2) this factors by a unique $\pi_{i}, 1 \leq i \leq k$, through

$$
f_{i}: A_{i} \rightarrow(\vec{A}-\circ \iota) \text {. }
$$

By (a5) we obtain $g: ! \vec{A} \rightarrow \vec{B}_{i}$, and by (a3) and the universal property of the product we obtain

$$
g=\left\langle g_{1}, \ldots, g_{q_{i}}\right\rangle^{\dagger} .
$$

We replace the continuity postulates appropriate for PCF by a finiteness axiom. We stipulate that each morphism $f: A \rightarrow B$ in $\mathcal{C}$ has a norm $\|f\|_{A, B} \in \mathbb{N}$, such that

$$
f=\mathbf{C}_{i}\left(g_{1}, \ldots, g_{q_{i}}\right) \Rightarrow \sup _{j=1, \ldots, q_{i}}\left\|g_{j}\right\|<\|f\| .
$$

Theorem 9.2 (Definability) Let $T$ be a simple type built from $\iota$. Then every $f: 1 \rightarrow \llbracket T \rrbracket$

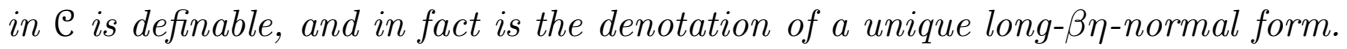

Proof By complete induction on $\|f\|$. If $T=\widetilde{T} \Rightarrow \iota$ and $f=\mathbf{C}_{i}\left(g_{1}, \ldots, g_{q_{i}}\right)$, then

$$
f=\llbracket \lambda \tilde{x}: \widetilde{T} \cdot x_{i}\left(M_{1} \tilde{x}\right) \cdots\left(M_{q_{i}} \tilde{x}\right) \rrbracket
$$

where $g_{j}=\llbracket M_{j} \rrbracket, 1 \leq j \leq q_{i}$. 
Examples The intended examples are the versions of [AJM96, HO96, McC96a] in which only total, compact strategies are included. Strictness of a strategy $\sigma: A \rightarrow B$ means that it responds to the opening move by moving in $A$. The interpretation of $\iota$ is as the game with a single move, which is an Opponent question.

The above result is essentially a characterization of the free cartesian closed category generated by the one-object one-morphism category. More general characterization results can probably be developed along similar lines.

Since under the Curry-Howard isomorphism [GLT89] the pure simply-typed $\lambda$-calculus corresponds to minimal implicational logic, this result has some relevance for Proof Theory. An interesting contrast with the full completeness results proved for Multiplicative Linear Logic in [AJ94 and a number of other subsequent works is that various notions of "uniformity", dinaturality etc. play an important role in those results, but do not arise here.

Universality In AJM96, HO96, a stronger result than Full Abstraction is proved, namely Universality, i.e. that all recursive strategies are definable in PCF; or, equivalently, that the model consisting of just the recursive strategies has all its elements definable. This is the strongest possible definability result, and is closely related to the notion of "Logical Full Abstraction" introduced by Longley and Plotkin in LP96, as shown loc. cit.

The axiomatic methods developed in the present paper can be extended to yield this stronger result. We briefly sketch the necessary extensions. Firstly, we take our sequential categories to be enriched over enumerated sets [AL91 rather than just pointed sets. All the isomorphisms required in the axioms have then to be given effectively. This leads to an effective version of the Decomposition Theorem as in [AJM96]. The development then proceeds exactly as in AJM96]. That is, universal terms are defined in PCF, from which the definability of all strategies follows directly.

Ackowledgements The research described in this paper was supported by the U.K. EPSRC grant "Foundational Structures for Computing Science". I am grateful to Guy McCusker and the two anonymous referees for their comments on the preliminary version of this paper.

\section{References}

[AJ94] S. Abramsky and R. Jagadeesan. Games and full completeness for multiplicative linear logic. Journal of Symbolic Logic, 59(2):543-574, 1994.

[AJM96] S. Abramsky, R. Jagadeesan, and P. Malacaria. Full abstraction for PCF. Submitted for publication, 1996.

[AL91] A. Asperti and G. Longo. Categories, Types and Structures. MIT Press, 1991.

[AM95] S. Abramsky and G. McCusker. Games and full abstraction for the lazy $\lambda$-calculus. In Tenth Annual Symposium on Logic in Computer Science, pages 234-243, 1995.

[AM97a] S. Abramsky and G. McCusker. Call-by-value games. In Computer Science Logic: CSL '9\%, 1997.

[AM97b] S. Abramsky and G. McCusker. Full abstraction for idealized algol with passive expressions. submitted for publication, 1997. 
[AM97c] S. Abramsky and G. McCusker. Linearity, sharing and state. In P. O'Hearn and R. D. Tennent, editors, Algol-like Languages, pages 297-329. Birkhauser, 1997.

[BCL86] G. Berry, P.-L. Curien, and J.-J. Lévy. Full abstraction for sequential languages: the state of the art. In M. Nivat and J. C. Reynolds, editors, Algebraic Semantics, pages 89-132. Cambridge University Press, 1986.

[Bie95] G. Bierman. What is a categorical model of intuitionistic linear logic? In International Conference onTyped Lambda Calculi and Applications. Springer-Verlag, 1995. Lecture Notes in Computer Science.

[Bra97] T. Brauner. A simple adequate categorical model for PCF. In Proceedings of Third International Conference on Typed Lambda Calculi and Applications, volume 1210 of Lecture Notes in Computer Science. Springer-Verlag, 1997.

[Cro94] R. Crole. Categories for Types. Cambridge University Press, 1994.

[Cur93] P.-L. Curien. Categorical Combinators, Sequential Algorithms and Functional Programming. Birkhauser, 1993.

[DHR96] V. Danos, H. Herbelin, and L. Regnier. Games and abstract machines. In International Symposium on Logic in Computer Science, 1996.

[DP90] B. Davey and H. Priestley. Introduction to Lattices and Order. Cambridge University Press, 1990.

[Fio96] M. Fiore. Axiomatic domain theory in categories of partial maps. Cambridge University Press, 1996.

[FP94] M. Fiore and G. Plotkin. An axiomatization of computationally adequate domain theoretic models of FPC. In Ninth Annual IEEE Symposium on Logic in Computer Science, pages 92-102, 1994.

[Gir87] J.-Y. Girard. Linear logic. Theoretical Computer Science, 50:1-102, 1987.

[GLT89] J.-Y. Girard, Y. Lafont, and P. Taylor. Proofs and Types. Cambridge University Press, 1989.

[HJ97] H. Hu and A. Joyal. Coherence completion of categories. Submitted for publication, 1997.

[HO96] M. Hyland and C.H. L. Ong. On full abstraction for PCF. Submitted for publication, 1996.

[HY97] K. Honda and N. Yoshida. Game-theoretic analysis of call-by-value computation. In Automata, Languages and Programming: 24th International Colloquium, ICALP '97, Lecture Notes in Computer Science, pages 225-236. Springer-Verlag, 1997.

[Hyl91] M. Hyland. First steps in synthetic domain theory. In Category Theory. SpringerVerlag, 1991. Lecture Notes in Mathematics vol. 1488.

[Joy95a] A. Joyal. Free bicomplete categories. Math. Rep., Acad. Sci. Canada, 17(5):219225, October 1995. 
[Joy95b] A. Joyal. Free lattices, communication and money games. In Proceedings of the 10th International Congress on Logic, Methodology and Philosophy of Science, 1995.

[Koc81] A. Kock. Synthetic Differential Geometry. Cambridge University Press, 1981.

[Lai97] J. G. Laird. Full abstraction for functional languages with control. In Twelfth Annual IEEE Symposium on Logic in Computer Science, pages 58-64, 1997.

[LP96] J. Longley and G. Plotkin. Logical full abstraction and PCF. To appear in: Proceedings ofTbilisi Symposium on Logic, Language and Computation, SiLLI/CSLI, 1996.

[Man76] E. Manes. Algebraic Theories. Springer-Verlag, 1976.

[McC96a] G. McCusker. Games and Full Abstraction for a functional metalanguage with recursive types. $\mathrm{PhD}$ thesis, Imperial College, University of London, 1996. to appear.

[McC96b] G. McCusker. Games and full abstraction for FPC. In International Symposium on Logic in Computer Science, 1996.

[Mil75] R. Milner. Processes: a mathematical model of computing agents. In Logic Colloquium '73, pages 157-173. North Holland, 1975.

[Mil77] R. Milner. Fully abstract models of typed $\lambda$-calculi. Theoretical Computer Science, 4:1-22, 1977.

[Mil92] R. Milner. Functions as processes. Mathematical Structures in Computer Science, 2(2):119-142, 1992.

[MMP95] A. Mifsud, R. Milner, and J. Power. Control structures. In Tenth Annual Symposium on Logic in Computer Science, pages 188-198, 1995.

[MOM91] N. Marti-Oliet and J. Meseguer. From Petri nets to linear logic via categories: a survey. International Journal on Foundations of Computer Science, 2(4):297-399, 1991.

[Nic94] H. Nickau. Hereditarily sequential functionals. In Proceedings of the Symposium on Logical Foundations of Computer Science: Logic at St. Petersburg, volume 813 of Lecture Notes in Computer Science. Springer-Verlag, 1994.

[Ong95] C.-H. L. Ong. Correspondence between operational and denotational semantics: the full abstraction problem for PCF. In S. Abramsky, D. Gabbay, and T. S. E. Maibaum, editors, Handbook of Logic in Computer Science, volume 4, pages 270356. Oxford University Press, 1995.

[Plo77] G. Plotkin. LCF considered as a programming language. Theoretical Computer Science, 5:223-255, 1977.

[See87] R. A. G. Seeley. Linear logic, *-autonomous categories and cofree coalgebras. In Category theory, computer science and logic. American Mathematical Society, 1987.

[Wal92] R. Walters. Categories and Computer Science. Cambridge University Press, 1992. 